\title{
Concomitants of Generalized Order Statistics for a Bivariate Weibull Distribution
}

\author{
Saman Hanif Shahbaz \\ Department of Statistics, Faculty of Sciences, \\ King Abdulaziz University, Jeddah, Saudi Arabia \\ saman.shahbaz17@gmail.com \\ Muhammad Qaiser Shahbaz \\ Department of Statistics, Faculty of Sciences \\ King Abdulaziz University, Jeddah, Saudi Arabia \\ qshahbaz@gmail.com
}

\begin{abstract}
In this paper we have studied the distribution of $r$-th concomitant and joint distribution of $r$-th and $s$-th concomitant of generalized order statistics for a bivariate Weibull distribution. We have derived the expression for single and product moments. Numerical study has also been conducted to see the behavior of mean of concomitants for selected values of the parameters.
\end{abstract}

Keywords: Concomitants, Generalized Order Statistics, Bivariate Weibull distribution.

\section{Introduction}

A bivariate Weibull distribution is defined by Hanif Shahbaz and Ahmad (2009) and by Ahsanullah et al. (2010) as a compound distribution of two Weibull random variables. The density function of bivariate Weibull distribution defined by Hanif Shahbaz and Ahmad (2009) is

$$
\begin{gathered}
f(x, y)=\beta \phi(x) \alpha_{1} \alpha_{2} x^{\alpha_{1}-1} y^{\alpha_{2}-1} \exp \left[-\left\{\beta x^{\alpha_{1}}+\phi(x) y^{\alpha_{2}}\right\}\right] ; \\
\alpha_{1}>0, \alpha_{2}>0, \beta>0, \phi(x)>0, x>0, y>0,
\end{gathered}
$$

where $\phi(x)$ is any positive real function of $X$. Ahsanullah et al. (2010) have studied the distribution (1.1) for $\phi(x)=x^{\alpha_{1}}$. The distribution in that case is given as

$$
f(x, y)=\beta \alpha_{1} \alpha_{2} x^{2 \alpha_{1}-1} y^{\alpha_{2}-1} \exp \left[-x^{\alpha_{1}}\left(\beta+y^{\alpha_{2}}\right)\right] ; \beta>0, \alpha_{1}>0, \alpha_{2}>0, x>0, y>0 .
$$

Distribution (1.1) can be studied for other choices of $\phi(x)$. The distribution (1.2) has been study in context of order statistics and record values by Ahsanullah et al. (2010) and in context of order statistics by Hanif Shahbaz et al. (2011).

The generalized order statistics (gos) has been defined by Kamps (1995) as a unified model for ordered random variables. Kamps (1995) has argued that the quantities $X_{r: n, m, k}$ are called gos if their joint distribution is given as

$$
\begin{aligned}
f_{1, n, n, m, k}\left(x_{1}, x_{2}, \quad, x_{n}\right)= & k\left(\prod_{j=1}^{n-1} \gamma_{j}\right)\left\{1-F\left(x_{n}\right)\right\}^{k-1} f\left(x_{n}\right) \\
& \times\left[\prod_{j=1}^{n-1}\left\{1-F\left(x_{i}\right)^{m}\right\} f\left(x_{i}\right)\right] ;
\end{aligned}
$$


where $n$ is sample size, $m$ and $k$ are parameters of the model and quantities $\gamma_{j}$ are given as $\gamma_{j}=k+(n-r)(m+1)$. The density function of a single gos is given by Kamps (1995) as

$$
f_{r: n, m, k}(x)=\frac{C_{r-1}}{(r-1) !} f(x)\{1-F(x)\}^{\gamma_{r}-1} g_{m}^{r-1}[F(x)],
$$

where $C_{r-1}=\prod_{j=1}^{r} \gamma_{j} ; r=1,2, \quad, n$, and

$$
g_{m}(x)=h_{m}(x)-h_{m}(0)= \begin{cases}{\left[1-(1-x)^{m+1}\right] /(m+1) ;} & m \neq-1 \\ -\ln (1-x) & m=-1 .\end{cases}
$$

We also have

$$
h_{m}(x)= \begin{cases}-(1-x)^{m+1} /(m+1) ; & m \neq-1 \\ -\ln (1-x) & m=-1 .\end{cases}
$$

Kamps (1995) has further shown that the joint density function of two GOS $X_{r: n, m, k}$ and $X_{s: n, m, k}$ for $r<s$ is given as

$$
\begin{aligned}
f_{r, s: n, m, k}\left(x_{1}, x_{2}\right)= & \frac{C_{s-1}}{(r-1) !(s-r-1) !} f\left(x_{1}\right) f\left(x_{2}\right)\left\{1-F\left(x_{1}\right)\right\}^{m} g_{m}^{r-1}\left\{F\left(x_{1}\right)\right\} \\
& \times\left\{1-F\left(x_{2}\right)\right\}^{\gamma_{s}-1}\left[h_{m}\left\{F\left(x_{2}\right)\right\}-h_{m}\left\{F\left(x_{1}\right)\right\}\right]^{s-r-1} ;-\infty<x_{1}<x_{2}<\infty .
\end{aligned}
$$

The density functions of GOS given in (1.4) and (1.5) provide several models of ordered random variables as special case. Specifically for $m=0$ and $k=1$ the model reduces to Ordinary Order Statistics as given by David and Nagaraja (2003). Also for $m=-1$ we obtain $k$ th upper record values introduced by Chandler (1952). Other models like fractional order statistics given by Stigler (1977), sequential order statistics etc. can also be obtained for various values of the parameters involved. Other special cases of gos can be seen in Shahbaz et al. (2017).

Sometime it happen that a sample is available from a bivariate distribution, say $F(x, y)$, and sample is arranged with respect to one of the variable, say $X$. The other variable, $Y$, is shuffled alongside the variable $X$ and is called the concomitant of $X$. When sample is arranged using order statistics then we have concomitants of order statistics and is discussed in David and Nagaraja (2003). Ahsanullah (1995) has discussed concomitants of record values. The concomitants of gos has been discussed by Ahsanullah and Nevzorov (2001) and by Shahbaz et al. (2017). The density function of $r$ th concomitant of gos is given as

$$
f_{[r: n, m, k]}(y)=\int_{-\infty}^{\infty} f(y \mid x) f_{r: n, m, k}(x) d x
$$

where $f(y \mid x)$ is conditional distribution of $Y$ given $X=x$ and $f_{r: n, m, k}(x)$ is defined in (1.4). The joint distribution of two concomitants is given as

$$
f_{[r, s: n, m, k]}\left(y_{1}, y_{2}\right)=\int_{-\infty}^{\infty} \int_{-\infty}^{x_{2}} f\left(y_{1} \mid x_{1}\right) f\left(y_{2} \mid x_{2}\right) f_{r, s: n, m, k}\left(x_{1}, x_{2}\right) d x_{1} d x_{2},
$$

where $f_{r, s: n, m, k}\left(x_{1}, x_{2}\right)$ is given in (1.5). 
Various authors have studied concomitants of gos. Concomitants of gos for Gumbel Bivariate Exponential distribution has been studied by Ahsanullah and Beg (2006). Further Beg and Ahsanullah (2008) has studied concomitants of GOS for Gumbel bivariate family of distributions. Nayabuddin (2013) has studied concomitants of GOS for bivariate Lomax distribution. Hanif Shahbaz and Shahbaz (2016) have studied the concomitants of gos for a bivariate exponential distribution.

In this paper we have obtained the distribution of the concomitants of upper record statistics for Bivariate Pseudo-Weibull distribution. Firstly, we have defined the Bivariate Pseudo-Weibull distribution in the following section.

\section{Bivariate Pseudo-Weibull Distribution}

The bivariate pseudo Weibull distribution has been defined by Hanif Shahbaz and Ahmad (2009) as compound distribution of Weibull random variables. The density function of bivariate Weibull distribution is given in (1.2). From the density function we can readily see that the marginal density function of $X$ is

$$
f(x)=\beta \alpha_{1} x^{\alpha_{1}-1} \exp \left(-\beta x^{\alpha_{1}}\right) ; \beta>0, \alpha_{1}>0, x>0 .
$$

The conditional distribution of $Y$ given $X=x$ is

$$
f(y \mid x)=\alpha_{2} x^{\alpha_{1}} y^{\alpha_{2}-1} \exp \left(-x^{\alpha_{1}} y^{\alpha_{2}}\right) ; \alpha_{1}>0, \alpha_{2}>0, x>0, y>0 .
$$

The marginal and conditional distributions are useful in studying the distribution of concomitants of gos for bivariate Weibull distribution.

In the following section the distribution of concomitant of record statistics has been derived for (1.2).

\section{Distribution of $\boldsymbol{r}$-th Concomitant and its Properties}

The Bivariate Pseudo-Weibull distribution has been given in (1.1) and (1.2). In this section the distribution of $r$-th concomitants of gos for Bivariate Pseudo-Weibull distribution, given in (1.2), has been obtained.

In order to obtain the distribution of concomitant of gos we first need the distribution of $r$ th $g o s$ for the marginal distribution of $X$ given in (2.1). The distribution of $g$ os for $X$ can be obtained by using (1.4). For this we first see that

$$
F(x)=1-\exp \left(-\beta x^{\alpha_{1}}\right) ; \beta>0, \alpha_{1}>0, x>0 \text {. }
$$

Also

$$
g_{m}[F(x)]=\frac{1}{m+1}\left[1-\{1-F(x)\}^{m+1}\right]=\frac{1}{m+1}\left[1-\exp \left\{-(m+1) \beta x^{\alpha_{1}}\right\}\right]
$$

So

$$
\begin{aligned}
g_{m}^{r-1}[F(x)] & =\frac{1}{(m+1)^{r-1}}\left[1-\exp \left\{-(m+1) \beta x^{\alpha_{1}}\right\}\right]^{r-1} \\
& =\frac{1}{(m+1)^{r-1}} \sum_{i=0}^{r-1}(-1)^{i}\left(\begin{array}{c}
r-1 \\
i
\end{array}\right) \exp \left\{-(m+1) \beta i x^{\alpha_{1}}\right\} .
\end{aligned}
$$


Now, using (2.1) and (3.1) in (1.4), the distribution of $r$ th $g o s$ for $X$ is

$$
\begin{aligned}
& f_{r: n, m, k}(x)=\frac{C_{r-1}}{(r-1) !} f(x)\{1-F(x)\}^{\gamma_{r}-1} g_{m}^{r-1}[F(x)] \\
& f_{r: n, m, k}(x)=\frac{C_{r-1}}{(r-1) !} \beta \alpha_{1} x^{\alpha_{1}-1} \exp \left(-\beta x^{\alpha_{1}}\right) \exp \left\{-\beta x^{\alpha_{1}-1}\left(\gamma_{r}-1\right)\right\} \\
& \quad \times \frac{1}{(m+1)^{r-1}} \sum_{i=0}^{r-1}(-1)^{i}\left(\begin{array}{c}
r-1 \\
i
\end{array}\right) \exp \left\{-(m+1) \beta i x^{\alpha_{1}}\right\} \\
& \text { or } \quad f_{r: n, m, k}(x)=\frac{C_{r-1}}{(r-1) !(m+1)^{r-1}} \sum_{i=0}^{r-1}(-1)^{i}\left(\begin{array}{c}
r-1 \\
i
\end{array}\right) \beta \alpha_{1} x^{\alpha_{1}-1} \exp \left(-\beta w_{1} x^{\alpha_{1}}\right),
\end{aligned}
$$

where $w_{1}=\left\{(m+1) i+\gamma_{r}\right\}$.

The conditional distribution of $Y$ given $X$ is given in (2.2). Now using (2.2) and (3.2) in (1.6), the distribution of $r$ th concomitant of gos for bivariate Weibull distribution is

$$
\begin{gathered}
f_{[r: n, m, k]}(y)=\int_{0}^{\infty} \alpha_{2} x^{\alpha_{1}} y^{\alpha_{2}-1} \exp \left(-x^{\alpha_{1}} y^{\alpha_{2}}\right) \frac{C_{r-1}}{(r-1) !(m+1)^{r-1}} \\
\quad \times \sum_{i=0}^{r-1}(-1)^{i}\left(\begin{array}{c}
r-1 \\
i
\end{array}\right) \beta \alpha_{1} x^{\alpha_{1}-1} \exp \left(-\beta w_{1} x^{\alpha_{1}}\right) d x \\
\text { or } \quad f_{[r: n, m, k]}(y)=\frac{C_{r-1}}{(r-1) !(m+1)^{r-1}} \sum_{i=0}^{r-1}(-1)^{i}\left(\begin{array}{c}
r-1 \\
i
\end{array}\right) \frac{\beta \alpha_{2} y^{\alpha_{2}-1}}{\left(y^{\alpha_{2}}+\beta w_{1}\right)^{2}} ; y>0 .
\end{gathered}
$$

The distribution of concomitants for special cases when sample is available from a bivariate Weibull distribution can be obtained from (3.3) by using specific values of the parameters involved.

The $r$-th moment of the distribution given in (3.3) is obtained as:

$$
\mu_{[r: n, m, k]}^{p}=\frac{C_{r-1}}{(r-1) !(m+1)^{r-1}} \sum_{i=0}^{r-1}(-1)^{i}\left(\begin{array}{c}
r-1 \\
i
\end{array}\right) \int_{0}^{\infty} y^{p} \frac{\beta \alpha_{2} y^{\alpha_{2}-1}}{\left(y^{\alpha_{2}}+\beta w_{1}\right)^{2}} d y,
$$

which after simplifications become

$$
\mu_{[r: n, m, k]}^{p}=\frac{\beta p C_{r-1} \Gamma\left(p / \alpha_{1}\right) \Gamma\left(1-p / \alpha_{2}\right)}{\alpha_{2}(r-1) !(m+1)^{r-1}} \sum_{i=0}^{r-1}(-1)^{i}\left(\begin{array}{c}
r-1 \\
i
\end{array}\right)\left(\beta w_{1}\right)^{p / \alpha_{2}-1}
$$

which exist for $p<\alpha_{2}$. We can see that the moment expression given in (3.5) reduces to expression for moments of concomitants of order statistics given by Shahbaz et al. (2009) for $m=0$ and $k=1$. The table of means for $m=2, k=2$ and for various values of $n, \beta$ and $\alpha_{2}$ is given below. 
Table 1: Mean of gos for Selected Values of Parameters

\begin{tabular}{|c|c|c|c|c|c|c|c|c|c|c|c|c|}
\hline \multirow[b]{2}{*}{$\beta$} & \multirow[b]{2}{*}{$\alpha_{2}$} & \multirow[b]{2}{*}{$n$} & \multicolumn{10}{|c|}{$r$} \\
\hline & & & 1 & 2 & 3 & 4 & 5 & 6 & 7 & 8 & 9 & 10 \\
\hline \multirow{6}{*}{1.5} & \multirow{6}{*}{2.5} & 5 & 4.499 & 16.873 & 23.200 & 13.533 & 2.538 & & & & & \\
\hline & & 6 & 4.947 & 23.499 & 44.060 & 40.388 & 17.670 & 2.650 & & & & \\
\hline & & 7 & 5.345 & 30.737 & 72.999 & 91.249 & 62.734 & 21.957 & 2.745 & & & \\
\hline & & 8 & 5.706 & 38.518 & 110.739 & 175.338 & 164.379 & 90.408 & 26.369 & 2.825 & & \\
\hline & & 9 & 6.038 & 46.792 & 157.923 & 302.685 & 359.439 & 269.579 & 123.557 & 30.889 & 2.896 & \\
\hline & & 10 & 6.345 & 55.518 & 215.131 & 484.045 & 695.815 & 661.024 & 413.140 & 162.305 & 35.504 & 2.959 \\
\hline \multirow{6}{*}{2.0} & \multirow{6}{*}{3.0} & 5 & 3.579 & 13.422 & 18.455 & 10.765 & 2.018 & & & & & \\
\hline & & 6 & 3.893 & 18.494 & 34.675 & 31.786 & 13.906 & 2.086 & & & & \\
\hline & & 7 & 4.168 & 23.968 & 56.924 & 71.155 & 48.919 & 17.122 & 2.140 & & & \\
\hline & & 8 & 4.414 & 29.794 & 85.658 & 135.625 & 127.148 & 69.931 & 20.397 & 2.185 & & \\
\hline & & 9 & 4.637 & 35.934 & 121.276 & 232.446 & 276.029 & 207.022 & 94.885 & 23.721 & 2.224 & \\
\hline & & 10 & 4.841 & 42.358 & 164.137 & 369.308 & 530.881 & 504.337 & 315.211 & 123.833 & 27.088 & 2.257 \\
\hline \multirow{6}{*}{2.5} & \multirow{6}{*}{3.5} & 5 & 3.018 & 11.318 & 15.563 & 9.078 & 1.702 & & & & & \\
\hline & & 6 & 3.258 & 15.477 & 29.019 & 26.601 & 11.638 & 1.746 & & & & \\
\hline & & 7 & 3.466 & 19.927 & 47.326 & 59.158 & 40.671 & 14.235 & 1.779 & & & \\
\hline & & 8 & 3.649 & 24.628 & 70.805 & 112.108 & 105.101 & 57.806 & 16.860 & 1.806 & & \\
\hline & & 9 & 3.813 & 29.550 & 99.732 & 191.153 & 226.994 & 170.246 & 78.029 & 19.507 & 1.829 & \\
\hline & & 10 & 3.962 & 34.672 & 134.353 & 302.294 & 434.548 & 412.820 & 258.013 & 101.362 & 22.173 & 1.848 \\
\hline
\end{tabular}

From above table we can see that the expected values shows an interesting trend. For even values of $n$, the mean increases for $1 \leq r \leq(n / 2)$ and decreases for $(n / 2)<r \leq n$. For odd values of $n$, the mean increases for $1 \leq r \leq(n+1) / 2$ and decreases for $(n+1) / 2<r \leq n$. It can be further seen that for fixed value of $n$, the mean decreases with increase in $\beta$ and $\alpha_{2}$.

The mean and the variance of the concomitants of gos for other values of parameters can also be tabulated.

The distribution function of $r$ th concomitant of gos for bivariate Weibull distribution is given as

$$
\begin{aligned}
F_{[r: n, m, k]}(y) & =\frac{C_{r-1}}{(r-1) !(m+1)^{r-1}} \sum_{i=0}^{r-1}(-1)^{i}\left(\begin{array}{c}
r-1 \\
i
\end{array}\right) \int_{0}^{y} \frac{\beta \alpha_{2} y^{\alpha_{2}-1}}{\left(y^{\alpha_{2}}+\beta w_{1}\right)^{2}} d y \\
& =\frac{C_{r-1}}{(r-1) !(m+1)^{r-1}} \sum_{i=0}^{r-1}(-1)^{i}\left(\begin{array}{c}
r-1 \\
i
\end{array}\right) \frac{y^{\alpha_{2}}}{w_{1}\left(y^{\alpha_{2}}+\beta w_{1}\right)} .
\end{aligned}
$$

The hazard rate function for concomitant of gos for bivariate Weibull distribution can be easily written by using (3.3) and (3.5) as

$$
h_{[r: n, m, k]}(y)=\frac{\frac{C_{r-1}}{(r-1) !(m+1)^{r-1}} \sum_{i=0}^{r-1}(-1)^{i}\left(\begin{array}{c}
r-1 \\
i
\end{array}\right) \frac{\beta \alpha_{2} y^{\alpha_{2}-1}}{\left(y^{\alpha_{2}}+\beta w_{1}\right)^{2}}}{1-\frac{C_{r-1}}{(r-1) !(m+1)^{r-1}} \sum_{i=0}^{r-1}(-1)^{i}\left(\begin{array}{c}
r-1 \\
i
\end{array}\right) \frac{y^{\alpha_{2}}}{w_{1}\left(y^{\alpha_{2}}+\beta w_{1}\right)}} ; y>0 .
$$


The hazard rate function can be computed for given values of the parameters involved. In the following section we have obtained the joint distribution of two concomitants of gos for bivariate Weibull distribution.

\section{Joint Distribution of the Concomitants and Moments}

In this section we have derived the joint distribution of the concomitants of gos for bivariate Weibull distribution given in (1.2). The joint distribution is obtained by using expression (1.7). In order to obtain the joint distribution we first obtain the joint distribution of two gos by using (1.5) and is given as

$$
\begin{aligned}
f_{r, s: n, m, k}\left(x_{1}, x_{2}\right)= & \frac{C_{s-1}}{(r-1) !(s-r-1) !} \beta \alpha_{1} x_{1}^{\alpha_{1}-1} \exp \left(-\beta x_{1}^{\alpha_{1}}\right) \beta \alpha_{1} x_{2}^{\alpha_{1}-1} \exp \left(-\beta x_{2}^{\alpha_{1}}\right) \exp \left(-\beta m x_{1}^{\alpha_{1}}\right) \\
& \times \frac{1}{(m+1)^{r-1}} \sum_{i=0}^{r-1}(-1)^{i}\left(\begin{array}{c}
r-1 \\
i
\end{array}\right) \exp \left\{-\beta(m+1) i x_{1}^{\alpha_{1}}\right\} \exp \left\{-\beta x_{1}^{\alpha_{1}}\left(\gamma_{s}-1\right)\right\} \\
& \times \frac{1}{(m+1)^{s-r-1}}\left[\exp \left\{-\beta(m+1) x_{1}^{\alpha_{1}}\right\}-\exp \left\{-\beta(m+1) x_{2}^{\alpha_{1}}\right\}\right]^{s-r-1},
\end{aligned}
$$

which after simplification becomes

$$
\begin{aligned}
f_{r, s: n, m, k}\left(x_{1}, x_{2}\right)= & \frac{C_{s-1}}{(r-1) !(s-r-1) !(m+1)^{s-2}} \sum_{i=0}^{r-1} \sum_{j=0}^{s-r-1}(-1)^{i+j}\left(\begin{array}{c}
r-1 \\
i
\end{array}\right)\left(\begin{array}{c}
s-r-1 \\
j
\end{array}\right) \\
& \times \beta^{2} \alpha_{1}^{2} x_{1}^{\alpha_{1}-1} x_{2}^{\alpha_{1}-1} \exp \left(-\beta w_{2} x_{1}^{\alpha_{1}}\right) \exp \left(-\beta w_{3} x_{2}^{\alpha_{1}}\right) ; \quad 0<x_{1}<x_{2}<\infty,
\end{aligned}
$$

where $w_{2}=\{(m+1)(s-r-j+i)\} ; w_{3}=\left\{(m+1) j+\gamma_{s}\right\}$.

Using the distribution (4.1) and (2.2) in (1.5), the joint distribution of two concomitants of gos for bivariate Weibull distribution is

$$
\begin{aligned}
f_{[r, s: n, m, k]}\left(y_{1}, y_{2}\right) & =\int_{0}^{\infty} \int_{x_{1}}^{\infty} \alpha_{2} x_{1}^{\alpha_{1}} y_{1}^{\alpha_{2}-1} \exp \left(-x_{1}^{\alpha_{1}} y_{1}^{\alpha_{2}}\right) \alpha_{2} x_{2}^{\alpha_{1}} y_{2}^{\alpha_{2}-1} \exp \left(-x_{2}^{\alpha_{1}} y_{2}^{\alpha_{2}}\right) \\
& \times \frac{C_{s-1}}{(r-1) !(s-r-1) !(m+1)^{s-2}} \sum_{i=0}^{r-1} \sum_{j=0}^{s-r-1}(-1)^{i+j}\left(\begin{array}{c}
r-1 \\
i
\end{array}\right)\left(\begin{array}{c}
s-r-1 \\
j
\end{array}\right) \\
& \times \beta^{2} \alpha_{1}^{2} x_{1}^{\alpha_{1}-1} x_{2}^{\alpha_{1}-1} \exp \left(-\beta w_{2} x_{1}^{\alpha_{1}}\right) \exp \left(-\beta w_{3} x_{2}^{\alpha_{1}}\right) d x_{2} d x_{1} \\
f_{[r, s: n, m, k]}\left(y_{1}, y_{2}\right) & =\frac{C_{s-1}}{(r-1) !(s-r-1) !(m+1)^{s-2}} \sum_{i=0}^{r-1} \sum_{j=0}^{s-r-1}(-1)^{i+j}\left(\begin{array}{c}
r-1 \\
i
\end{array}\right)\left(\begin{array}{c}
s-r-1 \\
j
\end{array}\right) \\
& \times \beta^{2} \alpha_{1}^{2} \alpha_{2}^{2} \int_{0}^{\infty} x_{1}^{2 \alpha_{1}-1} y_{1}^{\alpha_{2}-1} \exp \left\{-x_{1}^{\alpha_{1}}\left(y_{1}^{\alpha_{2}}+\beta w_{2}\right)\right\} I\left(x_{2}\right) d x_{1}
\end{aligned}
$$

where $I\left(x_{2}\right)=\int_{x_{1}}^{\infty} x_{2}^{2 \alpha_{1}-1} y_{2}^{\alpha_{2}-1} \exp \left\{-x_{2}^{\alpha_{1}}\left(y_{2}^{\alpha_{2}}+\beta w_{3}\right)\right\} d x_{2}$

Using the transformation $x_{2}^{\alpha_{1}}\left(y_{2}^{\alpha_{2}}+\beta w_{3}\right)=t$ and simplifying we have

$$
I\left(x_{2}\right)=\frac{y_{2}^{\alpha_{2}-1}}{\left(y_{2}^{\alpha_{2}}+\beta w_{3}\right)^{2}}\left\{1+x_{1}^{\alpha_{1}}\left(y_{2}^{\alpha_{2}}+\beta w_{3}\right) x_{1}\right\} \exp \left\{-x_{1}^{\alpha_{1}}\left(y_{2}^{\alpha_{2}}+\beta w_{3}\right)\right\} .
$$


Using this in (4.2) we have

$$
\begin{aligned}
f_{[r, s: n, m, k]}\left(y_{1}, y_{2}\right) & =\frac{C_{s-1}}{(r-1) !(s-r-1) !(m+1)^{s-2}} \sum_{i=0}^{r-1} \sum_{j=0}^{s-r-1}(-1)^{i+j}\left(\begin{array}{c}
r-1 \\
i
\end{array}\right)\left(\begin{array}{c}
s-r-1 \\
j
\end{array}\right) \\
& \times \beta^{2} \alpha_{1} \alpha_{2}^{2} \frac{y_{1}^{\alpha_{2}-1} y_{2}^{\alpha_{2}-1}}{\left(y_{2}^{\alpha_{2}}+\beta w_{3}\right)^{2}} \int_{0}^{\infty} x_{1}^{2 \alpha_{1}-1}\left\{1+x_{1}^{\alpha_{1}}\left(y_{2}^{\alpha_{2}}+\beta w_{3}\right) x_{1}\right\} \\
& \times \exp \left[-x_{1}^{\alpha_{1}}\left\{y_{1}^{\alpha_{2}}+y_{2}^{\alpha_{2}}+\beta\left(w_{2}+w_{3}\right)\right\}\right] d x_{1}
\end{aligned}
$$

Simplifying, the joint density function of two concomitants of gos for bivariate Weibull distribution is

$$
\begin{aligned}
f_{[r, s: n, m, k]}\left(y_{1}, y_{2}\right) & =\frac{\beta^{2} \alpha_{1} \alpha_{2} C_{s-1}}{(r-1) !(s-r-1) !(m+1)^{s-2}} \sum_{i=0}^{r-1} \sum_{j=0}^{s-r-1}(-1)^{i+j}\left(\begin{array}{c}
r-1 \\
i
\end{array}\right) \\
& \times\left(\begin{array}{c}
s-r-1 \\
j
\end{array}\right) \frac{y_{1}^{\alpha_{2}-1} y_{2}^{\alpha_{2}-1}\left\{y_{1}^{\alpha_{2}}+3 y_{2}^{\alpha_{2}-1}+\beta\left(w_{2}+w_{3}\right)\right\}}{\left(y_{2}^{\alpha_{1}}+\beta w_{3}\right)^{2}\left\{y_{1}^{\alpha_{2}}+y_{2}^{\alpha_{2}}+\beta\left(w_{2}+w_{3}\right)\right\}^{3}} .
\end{aligned}
$$

The product moments can be numerically computed from (4.3).

\section{Conclusions and Recommendations}

In this paper we have studied the distribution of concomitants of generalized order statistics when a sample is available from a bivariate Weibull distribution. The study has been conducted when $\phi(x)=x^{\alpha_{1}}$. We have obtained the distribution of single concomitant and joint distribution of two concomitants. We have seen that the distribution of single concomitant of gos for bivariate Weibull distribution is weighted sum of Burr XII distributions. We have also seen that the mean of concomitants of gos increase with increase in the value of $r$ until a specific point and then starts decreasing. This study can be extended by using some other choices of $\phi(x)$.

\section{Acknowledgement}

The authors are thankful to anonymous reviewer for providing constructive comments which help improve the quality of the paper.

\section{References}

1. Ahsanullah, M. (1995). Record Statistics, Nova Science Publisher, New York.

2. Ahsanullah, M. and Beg, M. I. (2007). Concomitant of generalized order statistics in Gumbel bivariate Exponential distribution, J. Stat. Th. and App., Vol. 6, 118132.

3. Ahsanullah, M. and Nevzorov, V. B. (2001). Ordered Random Variables, Nova Science Publishers, USA.

4. Ahsanullah, M., Shahbaz, S., Shahbaz, M. Q. and Mohsin, M. (2010). Concomitants of Upper Record Statistics for Bivariate Pseudo-Weibull distribution, App. \& Applied Math., Vol. 5(10), 1379-1388. 
5. Beg, M.I. and Ahsanullah, M. (2008). Concomitants of generalized order statistics from Farlie-Gumbel-Morgensterm distributions. Statistical Methodology, 5, 1-20.

6. Chandler, K. N. (1952). The distribution and frequency of record values, J. Royal Statist. Soc. B, 14, $220-228$.

7. David, H. A. and Nagaraja, H. (2003). Order Statistics. 3rd Edn. John Wiley \& Sons, New York.

8. Hanif Shahbaz, S. and Ahmad, M. (2009). Concomitants of order statistics for Bivariate Pseudo-Weibull distribution, World App. Sci. J., Vol. 6(10), pp 14091412.

9. Hanif Shahbaz, S. and Shahbaz, M. Q. (2016). Concomitants of Generalized Order Statistics for a Bivariate Exponential Distribution, Pak. J. Stat \& OR, Vol. 12(2), 227-234.

10. Hanif Shahbaz, S., Shahbaz, M. Q. and Rafiq, A. (2011). Bivariate Concomitants of Order Statistics for Pseudo Weibull Distribution, Nonlinear Analysis Forum, Vol. 16, 157-161.

11. Kamps, U. (1995). A concept of generalized order statistics, J. Statist. Plann. Inference, 48, 1-23.

12. Nayabuddin (2013). Concomitants of generalized order statistics from bivariate Lomax distribution, ProbStat Forum, Vol. 6, 73-88.

13. Shahbaz M. Q., Ahsanullah, M. and Hanif Shahbaz, S., Al-Zahrani, B. (2017). Ordered Random Variables: Theory and Applications, Atlantis Press \& Springer.

14. Stigler, S. M. (1977). Fractional order statistics, with applications, J. Amer. Statist. Assoc. 72, 544-550. 